\title{
Zum Satz von Dirichlet
}

\begin{abstract}
Peter Thurnheer
Peter Thurnheer studierte an der ETH Zürich Mathematik und promovierte im Jahr 1979 bei K. Chandrasekharan. Danach arbeitete er in verschiedenen Funktionen an Gymnasien, Fachhochschulen und Universitäten, von Haiti über Paris, Zürich bis Trogen. Gegenwärtig ist er an der Pädagogischen Hochschule und an der ETH Zürich tätig, wo er seit über fünfzehn Jahren Grundvorlesungen in Analysis und Elementarmathematik hält.
\end{abstract}

\section{Einleitung, der Satz von Dirichlet}

Die Theorie der diophantischen Approximation beginnt mit der Frage, wie gut sich eine gegebene, reelle Zahl $\alpha$ durch rationale Zahlen approximieren, das heisst, wie klein sich $|\alpha-p / q|, p / q \in \mathbb{Q}$, in Funktion des Nenners $q$ machen lässt. Leicht umformuliert lautet die Frage: Wie klein kann der Abstand von $\alpha q, q \in \mathbb{Z}$, zur nächsten ganzen Zahl in Abhängigkeit von $q$ gemacht werden?

Sind $n$ reelle Zahlen $\alpha_{1}, \ldots, \alpha_{n}$ gegeben, so stehen am Anfang der Theorie zwei analoge, eng miteinander verknüpfte Fragen:

i) Wie klein kann in Abhängigkeit der ganzen Zahl $q$ das Maximum der Abstände von $\alpha_{j} q, j=1, \ldots, n$, zur nächsten ganzen Zahl gemacht werden?

ii) Wie klein kann für ganze Zahlen $q_{1}, \ldots, q_{n}$ und in Abhängigkeit von $q=$ $\max \left(\left|q_{1}\right|, \ldots,\left|q_{n}\right|\right)$, der Abstand von $\alpha_{1} q_{1}+\cdots+\alpha_{n} q_{n}$ zur nächsten ganzen Zahl gemacht werden?

In der Theorie der diophantischen Approximation beschäftigt man sich mit der Frage, wie gut sich eine reelle Zahl $\alpha$ durch rationale Zahlen annähern lässt. In Verallgemeinerung auf $n$ gegebene reelle Zahlen $\alpha_{1}, \ldots, \alpha_{n}$ ergibt sich damit die Frage, wie klein man für ganze Zahlen $q_{1}, \ldots, q_{n}$ in Abhängigkeit von $q=\max \left(\left|q_{1}\right|, \ldots,\left|q_{n}\right|\right)$ den Abstand von $\alpha_{1} q_{1}+\cdots+\alpha_{n} q_{n}$ zur nächsten ganzen Zahl $p$ machen kann. Nach einem Satz von P.G.L. Dirichlet lässt sich diese Frage effektiv beantworten. Im vorliegenden Beitrag geht es um eine Verschärfung des Dirichletschen Satzes im Hinblick auf einschränkende Bedingungen an die Lage der approximierenden Gitterpunkte $\left(q_{1}, \ldots, q_{n}\right) \in \mathbb{Z}^{n}$. 
Die Antworten auf diese grundlegenden, allgemeinen Fragen wurden 1842 von Dirichlet gegeben. Wir betrachten hier die zweite Problemstellung. Wir nehmen im Weitern immer an, die reellen Zahlen $\alpha_{1}, \ldots, \alpha_{n}$ seien gegeben. Für ein Element $\underline{x}=\left(x_{1}, \ldots, x_{n}\right) \in \mathbb{R}^{n}$ bezeichne $L(\underline{x})$ den Abstand von $\alpha_{1} x_{1}+\cdots+\alpha_{n} x_{n}$ zur nächsten ganzen Zahl, das heisst

$$
L(\underline{x})=\min _{p \in \mathbb{Z}}\left|\alpha_{1} x_{1}+\cdots+\alpha_{n} x_{n}+p\right| \quad \text { und sei } \quad|\underline{x}|=\max \left(\left|x_{1}\right|, \ldots,\left|x_{n}\right|\right) \text {. }
$$

Dann lautet der

Satz von Dirichlet. Seien $\alpha_{1}, \ldots, \alpha_{n}$ reelle Zahlen. Dann gibt es zu jedem $N>1$ einen Gitterpunkt $\underline{q} \in \mathbb{Z}^{n}$ mit

$$
1 \leq|\underline{q}|<N \quad \text { und } \quad L(\underline{q}) \leq 1 / N^{n} .
$$

Ineffektive Version des Satzes von Dirichlet. Zu jedem $n$-Tupel $\alpha_{1}, \ldots, \alpha_{n}$ reeller Zahlen gibt es unendlich viele Gitterpunkte $\underline{q} \in \mathbb{Z}^{n}$ mit

$$
L(\underline{q})<1 /|\underline{q}|^{n} .
$$

Einführungen in die Theorie der diophantischen Approximation findet man in [1] und [2].

\section{Verschärfungen unter Verzicht auf die Effektivität}

Verzichtet man im Satz von Dirichlet auf die Effektivität, so lässt sich mehr beweisen als die obige, unmittelbare Folgerung, indem die Ungleichung (2) verschärft werden kann. Dieses Problem wurde ausführlich untersucht. Es gilt zum Beispiel

Aussage 1. Zu jedem $n$-Tupel $\alpha_{1}, \ldots, \alpha_{n}$ reeller Zahlen gibt es unendlich viele Gitterpunkte $\underline{q} \in \mathbb{Z}^{n}$, für die gilt

$$
\begin{aligned}
& L(\underline{q})<c(n) /|\underline{q}|^{n} \quad \text { mit } \\
& \text { i) } c(1)=1 / \sqrt{5} \text { und } \text { ii) } c(n) \leq(n /(n+1))^{n}, n \geq 2 .
\end{aligned}
$$

Teil i) von Aussage 1 wurde 1891 bewiesen von Hurwitz, der auch gezeigt hat, dass die Konstante $1 / \sqrt{5}$ in diesem Fall bestmöglich ist ([1], [2]). $\mathrm{Zu}$ Teil ii) siehe man [2, Kap. II, §3].

Man kann aber die Effektivität im Satz von Dirichlet auch ersetzen durch eine Einschränkung in Bezug auf die Lage der approximierenden Gitterpunkte. In [3] wurden dazu Verallgemeinerungen der untenstehenden Aussage 2 bewiesen. Seien $\varepsilon$ und $\delta$ beliebige positive Zahlen. Für $\underline{x} \in \mathbb{R}^{n}, n \geq 2$, setzt $\operatorname{man} r(\underline{x})=\left(x_{1}^{2}+\cdots+x_{n-1}^{2}\right)^{1 / 2}$ und definiert das Teilgebiet

$$
\Phi(w)=\left\{\underline{x} \in \mathbb{R}^{n}|| x_{n} \mid \leq(1+\varepsilon)(r(\underline{x}))^{w} \operatorname{oder} r(\underline{x}) \leq 1\right\} \subset \mathbb{R}^{n} .
$$


Aussage 2. Zu jedem $n$-Tupel reeller Zahlen $\alpha_{1}, \ldots, \alpha_{n}, n \geq 2$, existieren unendlich viele Gitterpunkte $\underline{q} \in \mathbb{Z}^{n}$ mit

$$
\underline{q} \in \Phi\left(1+1 / n+1 / n^{2}\right) \quad \text { und } \quad L(\underline{q}) \leq(1+\delta) /|\underline{q}|^{n} .
$$

Die Einschränkung, zur Approximation nur Gitterpunkte aus $\Phi\left(1+1 / n+1 / n^{2}\right)$ zuzulassen, ist in folgendem Sinn nicht sehr wesentlich. Sei

$$
V(R)=\operatorname{Vol}\left(\Phi\left(1+1 / n+1 / n^{2}\right) \cap\left\{\underline{x} \in \mathbb{R}^{n}|| \underline{x} \mid \leq R\right\}\right) .
$$

Dann gilt offensichtlich

$$
V(R) /(2 R)^{n} \rightarrow 1 \quad \text { für } \quad R \rightarrow \infty
$$

Im Folgenden wird gezeigt, dass Aussage 2 richtig bleibt, wenn man $\Phi\left(1+1 / n+1 / n^{2}\right)$ ersetzt durch Gebiete $\Omega$, welche zwar von ganz anderer Form sind, aber in obigem Sinne eine wesentliche Einschränkung des $\mathbb{R}^{n}$ darstellen.

Zur Definition dieser Gebiete betrachten wir die Funktion $f(x)=(1+x)^{n+1} / x$. Sie hat auf $\mathbb{R}^{+}$genau ein relatives Minimum bei $x=1 / n$. Sei $\sigma=\sigma(n) \in(0,1)$ diejenige Zahl, für die gilt $f(\sigma)=f(1)=2^{n+1}$. Sei $d \leq 1 / \sigma$. Dann ist

$$
(1+x)^{n+1} / x \leq 2^{n+1} \quad \text { für alle } \quad x \in[1 / d, 1] .
$$

Mit $a=2^{(n+1) / n} d$ setzt man

$$
\Omega=\Omega(d)=\left\{\underline{x} \in \mathbb{R}^{n}\left|a^{j}<\right| \underline{x} \mid<2^{(n+1) / n} a^{j}, j=1,2, \ldots\right\} \subset \mathbb{R}^{n} .
$$

Satz. Zu jedem $n$-Tupel $\alpha_{1}, \ldots, \alpha_{n}, n \geq 2$, reeller Zahlen gibt es unendlich viele Gitterpunkte $\underline{q} \in \mathbb{Z}^{n}$ mit

$$
\underline{q} \in \Omega \quad \text { und } \quad L(\underline{q}) \leq 1 /|\underline{q}|^{n} .
$$

Bemerkung. Setzt man

$$
W(R)=\operatorname{Vol}\left(\Omega \cap\left\{\underline{x} \in \mathbb{R}^{n}|| \underline{x} \mid \leq R\right\}\right),
$$

so hat $W(R) /(2 R)^{n}$ relative Maxima bei $R=2^{(n+1) / n} a^{j}, j=1,2, \ldots$, und $W(R) /(2 R)^{n}$ kann durch eine geometrische Reihe abgeschätzt werden. Man erhält im Gegensatz zu (3)

$$
\limsup _{R \rightarrow \infty} W(R) /(2 R)^{n} \leq\left(1-1 / 2^{n+1}\right) /\left(1-1 / a^{n}\right)=\left(2^{n+1} d^{n}-d^{n}\right) /\left(2^{n+1} d^{n}-1\right)<1 .
$$

Für $n=2$ zum Beispiel, ist $\sigma=\sigma(2)<1 / 4$. Man kann $d=4$ wählen und erhält $\lim \sup _{R \rightarrow \infty} W(R) /(2 R)^{2}<0,882$.

Beweis des Satzes. Sei $q_{1}, q_{2}, \ldots$ eine Folge von Minimalpunkten, das heisst von Gitterpunkten so, dass mit $\left|\underline{q_{j}}\right|=N_{j}$ und $L\left(\underline{q}_{j}\right)=L_{j}$ für alle $j=1,2, \ldots$ gilt $N_{j}<N_{j+1}$ und $L(\underline{q}) \geq L_{j}$ für alle Gitterpunkte $\underline{q}$ mit $|\underline{q}|<N_{j}$. 
Auf Grund der effektiven Version des Satzes von Dirichlet ist dann

$$
L_{j} \leq 1 / N_{j+1}^{n}
$$

Man beweist den Satz indirekt, das heisst, man geht aus von der

Annahme. Es existieren höchstens endlich viele Gitterpunkte $\underline{q}$, die den Satz erfüllen.

$\mathrm{Zu}$ unendlich vielen Indizes $m$ gibt es dann einen Index $j=j(m)$ so, dass gilt $j(m) \rightarrow \infty$ mit $m \rightarrow \infty$ und

$$
2^{(n+1) / n} a^{j-1} \leq N_{m} \leq a^{j},
$$

sowie

$$
N_{m+1} \geq 2^{(n+1) / n} a^{j} .
$$

Mit $N_{m}=x a^{j}$ ist also $1 / d \leq x \leq 1$ wegen $2^{(n+1) / n} / a=1 / d$, so dass mit (4) gilt

$$
\left(a^{j}+N_{m}\right)^{n+1} / N_{m} \leq 2^{n+1} a^{n j} .
$$

Nach (5) und (6) ist zudem

$$
L_{m} \leq 1 / 2^{n+1} a^{n j}
$$

Es bezeichne $[z]$ den Ganzteil der reellen Zahl $z$.

Sei $b=\left[a^{j} / N_{m}\right]+1$. Für den Gitterpunkt $\underline{q}=\underline{q}(m)=b \underline{q}_{m}$ hat man

$$
a^{j}<|\underline{q}| \leq a^{j}+N_{m} \leq 2 a^{j}<2^{(n+1) / n} a^{j} .
$$

Also ist $q \underline{q} \in$ sowie mit (8) und (7)

$$
L(\underline{q}) \leq\left(a^{j}+N_{m}\right) / N_{m} 2^{n+1} a^{n j} \leq 1 /\left(a^{j}+N_{m}\right)^{n} \leq 1 /|\underline{q}|^{n} .
$$

Das bedeutet, dass der Gitterpunkt $\underline{q}(m)$ den Satz erfüllt, was für genügend grosses $m$ einen Widerspruch zur Annahme darstellt und damit den Satz beweist.

\section{Literatur}

[1] Schmidt, W.M.: Approximation to algebraic numbers. Enseign. Math. II, XVII (1971), 187-253.

[2] Schmidt, W.M.: Diophantine approximation. Lecture Notes in Math. 785, Springer-Verlag.

[3] Thurnheer, P.: On Dirichlets theorem concerning diophantine approximation. Acta Arith. LIV (1990).

Peter Thurnheer

Departement Mathematik

ETH Zürich

CH-8092 Zürich, Schweiz

e-mail: peter.thurnheer@math.ethz.ch 